\title{
Technè
}

La science au service de l'histoire de l'art et de la préservation des biens culturels

42 | 2015

Science et conservation

\section{Documenter pour l'avenir : la campagne de restauration des dessins d'Ingres du musée de Montauban (1946-1952)}

Documenting for the future: the restoration campaign of Ingres' drawings at the Musée de Montauban (1946-1952)

Natalie Coural, Laëtitia Desserrières et Florence Viguier-Dutheil

\section{(2) OpenEdition}

\section{Journals}

Édition électronique

URL : http://journals.openedition.org/techne/6762

DOI : $10.4000 /$ techne.6762

ISSN : 2534-5168

Éditeur

C2RMF

Édition imprimée

Date de publication : 1 décembre 2015

Pagination : 55-61

ISBN : 978-2-7118-6249-8

ISSN : $1254-7867$

\section{Référence électronique}

Natalie Coural, Laëtitia Desserrières et Florence Viguier-Dutheil, « Documenter pour l'avenir : la campagne de restauration des dessins d'Ingres du musée de Montauban (1946-1952) », Technè [En ligne], 42 | 2015, mis en ligne le 01 décembre 2015, consulté le 11 mars 2021. URL : http:// journals.openedition.org/techne/6762 ; DOI : https://doi.org/10.4000/techne.6762

\section{(ब) $(\Theta \Theta$}

La revue Technè. La science au service de l'histoire de l'art et de la préservation des biens culturels est mise à disposition selon les termes de la Licence Creative Commons Attribution - Pas d'Utilisation Commerciale - Pas de Modification 4.0 International. 


\section{Natalie Coural \\ Laëtitia Desserrières \\ Florence Viguier-Dutheil \\ Documenter pour l'avenir : la campagne de restauration des dessins d'Ingres du musée de Montauban (1946-1952)}

Documenting for the future: the restoration campaign of Ingres' drawings at the Musée de Montauban (1946-1952)

Résumé. En 1946, une inspection menée sur les dessins de Jean-Auguste-Dominique Ingres (1780-1867) conservés au musée Ingres de Montauban constate leur état de conservation préoccupant. Les æuvres sont envoyées à Paris pour examen et étude en vue de leur restauration et de leur remontage, parallèlement au réaménagement complet du musée. Depuis lors, les nombreux documents conservés permettent de mieux connaître les interventions entreprises, les réflexions qui les ont précédées et les méthodes utilisées. Cette documentation constitue aujourd'hui un apport important à la connaissance de la collection et à l'histoire de la restauration des dessins.

Mots-clés. Jean-Auguste-Dominique Ingres, Montauban, musée Ingres, Jacqueline Bouchot-Saupique, Clémence Duprat, Léon Lepeltier, Daniel Ternois, Jean Vergnet-Ruiz, Harvard, Fogg Art Museum, papier neutre.
Abstract. In 1946, the drawings of Jean-Auguste-Dominique Ingres (1780-1867) in the Musée Ingres, Montauban, were inspected and found to be in a worrying condition. The works were sent to Paris for further examination and study with a view to being restored and remounted, in tandem with the overall renovation of the museum. Since then, several documents kept in the archives have provided insight into the restoration processes undertaken, the logic behind them, and the methods used. Today these documents contribute to a better understanding of the collection and to the restoration of the drawings over the years.

Keywords. Jean-Auguste-Dominique Ingres, Montauban, Musée Ingres, Jacqueline Bouchot-Saupique, Clémence Duprat, Léon Lepeltier, Daniel Ternois, Jean Vergnet-Ruiz, Harvard, Fogg Art Museum, acid-free paper.
La restauration des œuvres sur papier a longtemps fait figure de «parent pauvre » comparée à celle de la peinture. À l'écart de la plupart des grandes polémiques, moins prestigieuse, elle s'est faite discrète : on ne parlait pas de restauration, mais de « raccommodage », de « lavage » et de « collage ». Aussi comptes rendus et analyses se sont mis en place plus tardivement.

Cependant, comme pour la peinture, la céramique ou la sculpture, la nécessité de documenter les œuvres pendant ou après l'intervention s'était affichée aux yeux de tous à l'époque révolutionnaire, avec le cas emblématique de la restauration du carton de Raphaël pour l'École d'Athènes, qui venait d'être saisi à l'Ambrosienne de Milan. Autre moment important, l'action menée au milieu du XIX ${ }^{\mathrm{e}}$ siècle par des hommes de musée - Jeanron, Villot ou Reiset -, que ce soit à Lille, au Louvre ou à Chantilly ${ }^{1}$.

Le cas de la campagne de restauration des dessins de Montauban apparaît extrêmement intéressant du point de vue de son abondant accompagnement documentaire ; il annonce les méthodes actuellement employées dans les institutions par l'ampleur de l'enquête qui fut alors menée.
En 1940, une partie des collections de peintures italiennes du Louvre est accueillie au musée Ingres. L'éloignement de près de 700 kilomètres n'empêche pas les liaisons entre Paris et Montauban : les œuvres déposées doivent être photographiées par Alexandre Séarl qui se déplace à plusieurs reprises, muni d'un sauf-conduit, passant la ligne de démarcation dans un camion transportant des milliers de plaques de verre ${ }^{2}$ Après l'invasion de la zone libre, le musée Ingres prend la précaution d'éloigner les 4500 dessins, légués par l'artiste à sa ville natale en 1867, en les entreposant provisoirement au château de La Poujade à Urval, en Dordogne, en juillet $1943^{3}$. En 1926, le conservateur Félix Bouisset avait déjà mentionné l'état de dégradation des dessins d'Ingres dans son ouvrage sur le musée ${ }^{4}$. L'artiste avait utilisé des papiers qui se sont mal conservés (supports acides, rongés par certaines encres). À cette mauvaise évolution matérielle, il faut ajouter les manipulations diverses du peintre sur sa collection et ses expérimentations à base de résines ou d'huiles pour rendre le papier transparent ${ }^{5}$. Enfin, la présentation permanente de plusieurs centaines de feuilles, depuis la mort de l'artiste jusqu'en 1939, avait achevé de fragiliser les dessins (fig. 1).

Natalie Coural, conservateur du patrimoine, C2RMF (natalie.coural@culture.gouv.fr). Laëtitia Desserrières, assistante, responsable de la collection de dessins, musée de l'Armée, Paris (laetitia.desserrieres@musee-armee.fr). Florence Viguier-Dutheil, conservateur en chef du patrimoine, directrice du musée Ingres de Montauban (FViguier@ville-montauban.fr). 


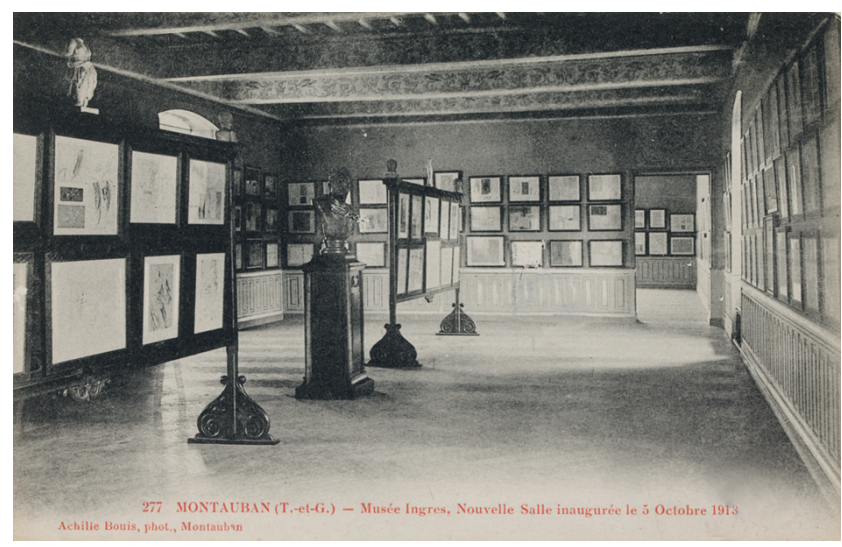

Fig. 1. Vue de la nouvelle salle des dessins ouverte en 1913 au $2^{\mathrm{e}}$ étage du musée grâce aux revenus de l'exposition Ingres de la galerie Georges Petit, tenue à Paris en 1911. Cette présentation conçue par Henry Lapauze était destinée à compléter les salles de dessins du $1^{\text {er }}$ étage et a permis d'exposer en permanence près de 2500 dessins au total jusqu'en 1939. (C) Montauban, musée Ingres, archives.

\section{Documenter pour mieux comprendre}

Au lendemain de la Libération, la plupart des musées de France sont confrontés à une situation critique. Des organismes centralisés sont alors créés, telle l'Inspection générale des musées de province, pour leur permettre de se reconstruire. Jean Vergnet-Ruiz ${ }^{6}$ (fig. 2), nommé à la tête de ce nouveau service, s'attache à redonner vie à ces musées, en privilégiant leur « couleur locale», leur histoire. Dès le mois de mai 1946, il met son expertise au service du musée Ingres. Pour organiser un cabinet des dessins «selon les dernières méthodes préconisées par la Bibliothèque nationale [et] le cabinet des Dessins du Louvre », il lui apparaît nécessaire de restaurer une partie de la collection de dessins et de les remonter selon des formats uniformisés pour en faciliter la manipulation ${ }^{7}$. Cette réorganisation inclut également le tri, le classement et l'inventaire complet des collections, ainsi qu'un nouvel aménagement muséographique permettant de présenter les œuvres dans les salles d'exposition ${ }^{8}$.

\section{Les acteurs : une équipe dynamique}

Jean Vergnet-Ruiz s'entoure d'une solide équipe parisienne : tout d'abord Georges-Henri Rivière, créateur du musée des Arts et Traditions populaires ${ }^{9}$, qui est membre du tout nouveau Conseil international des musées, l'ICOM, et bénéficie ainsi de l'aide d'un important réseau de chercheurs spécialisés. Du côté du musée du Louvre, Jacqueline Bouchot-Saupique ${ }^{10}$, nouvellement nommée conservateur au Cabinet des dessins, organise la campagne de restauration : elle choisit le restaurateur, Léon Lepeltier ${ }^{11}$, et l'encadreur, Émile Martin ${ }^{12}$. J. Bouchot-Saupique orchestre le travail, accompagnée dans ses tâches de surveillance des travaux par Clémence Duprat ${ }^{13}$, rattachée au corps de l'Inspection générale des musées de province. Cette dernière, méthodique, se déplace régulièrement à Montauban.

\section{Paris/Montauban : chronologie des interventions}

Les documents relatifs à cette campagne (rapports, pièces comptables, photographies, correspondance) permettent aujourd'hui de suivre les phases de réflexions et les interventions ${ }^{14}$. Une première inspection de Clémence Duprat et de Georges-Henri Rivière à Montauban a lieu du 2 au 19 décembre 1946, et aboutit à un rapport très détaillé. Par la suite, les échanges sont nombreux entre le musée Ingres et le musée du Louvre : les dessins sont régulièrement envoyés en caisses au musée parisien pour être photographiés, restaurés et enfin montés. Il est convenu que le restaurateur travaille au Louvre, dans un local mis à sa disposition. Malgré les informations qu'il a fournies pour l'installation de cet atelier et pour le matériel nécessaire aux traitements des œuvres, les mauvaises conditions dans lesquelles débute le travail sont évoquées à plusieurs reprises ${ }^{15}$. Il semblerait que Léon Lepeltier ait traité une partie des dessins dans son atelier parisien, comme le mentionnent certains de ses carnets ${ }^{16}$. Les 36 premiers dessins arrivent le 26 janvier 1947, puis les envois se succèdent entre 1948 et 1952. C'est à la suite des interventions sur le premier lot qu'est constituée une commission de spécialistes (experts, conservateurs, chimistes) pour le traitement et le montage des dessins, afin de s'accorder sur les meilleures méthodes de restauration et sur les matériaux les moins nocifs pour les œuvres. Cette commission se réunit à deux reprises, le 16 novembre 1948 et le 25 janvier 1949, et son travail met en lumière l'approche pluridisciplinaire qui a présidé aux décisions adoptées pour les différentes phases de traitements et de montage. La campagne de restauration est bien avancée en 1949 : une petite exposition à Paris, galerie André Weil, présente au mois de juin un choix de dessins, parmi lesquels les œuvres les plus importantes de la collection montalbanaise, comme l'Étude de mains et de pieds de la Vierge pour le Vou de Louis XIII et le Portrait de Madame Ingres ${ }^{17}$. Enfin, le travail s'achève en 1952 avec l'envoi de 133 œuvres.

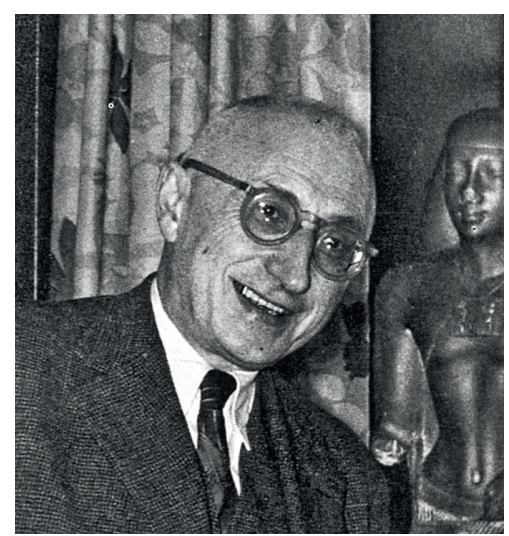

Fig. 2. Jean Vergnet-Ruiz, vers 1970. Photographe anonyme. (C) D.R. 


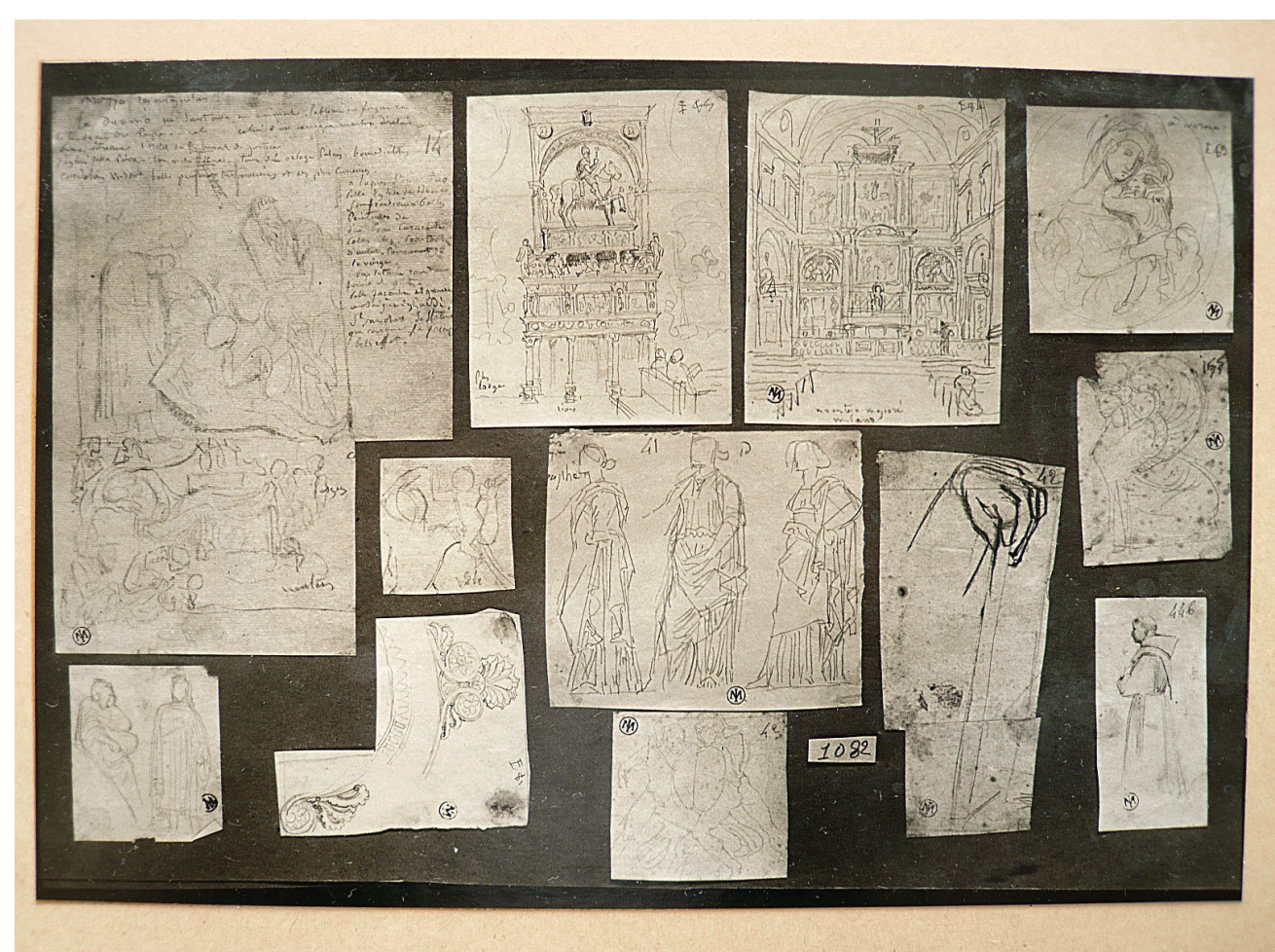

Fig. 3. Suite de divers croquis au crayon par Ingres, réunis sur une même planche. Montauban, musée Ingres. On reconnaît de gauche à droite et de haut en bas : Croquis et notes de voyages sur Arezzo et Lucques, MI 867.3999 ; Tombeau surmonté d'une statue équestre, MI 867.4066 ; Intérieur de l'église San Maurizio al Monasterio Maggiore, MI 867.4292 ; Vierge assise, MI 867.4065 ; Un enfant à califourchon sur le bras d'un homme nu, MI 867.2909 ; Frise antique, MI 867.3542 ; Longin (sa main gauche et sa tablette), MI 867.1017 ; Une sphinge (de profil à gauche), MI 867.3458 ; Deux personnages en costume du Moyen Âge, MI 867.4206 ; Détail d'encadrement, MI 867.4067 ; Deux guerriers grecs combattant, MI 867.3412; Moine debout (les bras croisés), MI 867.2952.

La plupart de ces dessins ont depuis été montés indépendamment sous passepartout.

Photographie d'Alexandre Séarl réalisée à l'arrivée au Louvre, avant traitement et démontage. (C) Paris, musée du Louvre, département des Peintures, Service d'études et de documentation/ Gilles Bastian.

\section{Documenter pour mieux connaître}

Les nombreux échanges et débats qui ont accompagné les prises de décision sont connus aujourd'hui à travers la documentation. Certaines pièces d'archives permettent de mieux comprendre quels produits ont été alors utilisés pour des interventions précises comme le nettoyage, d'autres renseignent sur une méthodologie d'ensemble appliquée à une importante collection.

\section{État des œuvres à leur arrivée à Paris : la campagne de photographies}

Une campagne de photographie est organisée de manière systématique à chaque fois que les dessins arrivent de Montauban, afin de préciser et de garder une trace de l'état de conservation des œuvres avant leur restauration. Les prises de vues sont réalisées par Alexandre Séarl. Les tirages montrent les œuvres sur leur carton de montage et les détériorations causées par le collage. Leur mise en parallèle avec les informations données dans les différents rapports rédigés lors des missions permet d'appréhender de manière complète les questions qui se sont alors posées. Ainsi, « presque tous les dessins sont complètement collés sur un bristol ou sur un carton d'une qualité inférieure. Ce montage défectueux a gaufré le feuillet, dont le détachement risque de mettre le dessin en péril », écrit Clémence Duprat ${ }^{18}$. Certains cartons comprennent jusqu'à douze dessins collés sur le même support, se chevauchant et sans ordre logique ${ }^{19}$ (fig. 3).

\section{Réflexions et évolution de ces réflexions en cours de traitement}

Les factures que le restaurateur adresse régulièrement au musée de Montauban précisent les interventions sur les œuvres : «Décollage, dressage, remargement et petites réparations ; carton pour montage, papier fort pour marges, fournitures diverses, emballage ; façon pour 26 passe-partout $^{20}$. » Certains dessins particulièrement fragiles, sur des supports très altérés, demandent d'autres types d'interventions et de nombreuses questions se posent. Par exemple, la commission s'interroge sur les dangers du désencadrement dans ces cas précis, et en vient à déplorer "l'absence de moyens techniques capables de retarder l'inévitable destruction d'un certain nombre d'entre eux, qui ne sont plus que "cendres ${ }^{21}$ ", telles une Étude pour Vénus Anadyomène $e^{22}$ (fig. 4). Elle se résout à conserver ces œuvres entre deux verres, «à titre documentaire et comme témoins d'un état de fait répréhensible au point de vue de la conservation des collections ${ }^{23}$ ». Ne pas intervenir parce que l'on ne sait pas traiter, comme on peut le lire dans les rapports, est aussi l'une des nouveautés de cette campagne. «C'est dans la recherche d'une meilleure 


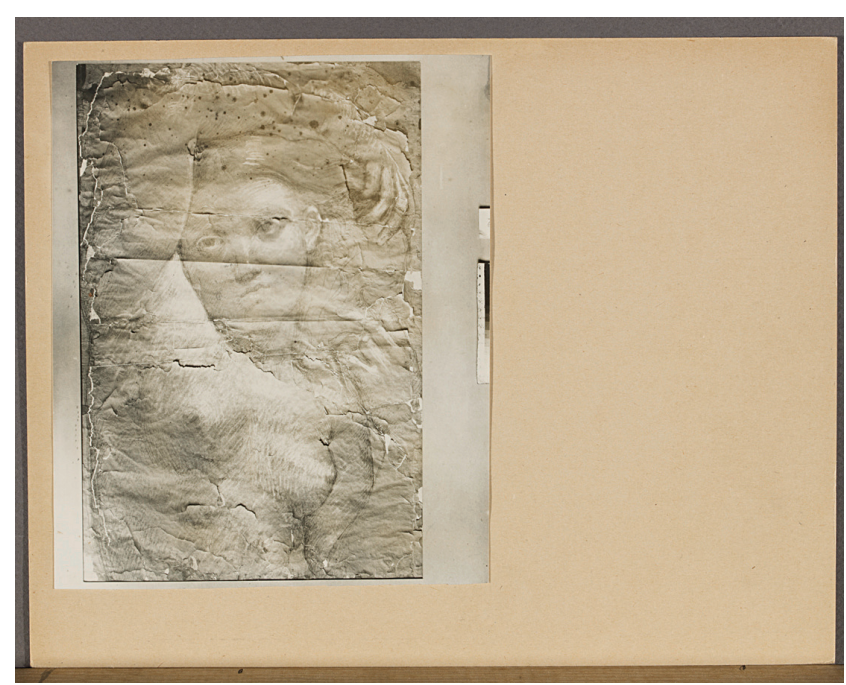

Fig. 4a. Ingres, Étude pour Vénus Anadyomène (MI 867.2313). Photographie d'Alexandre Séarl prise à l'arrivée du dessin au Louvre et collée ensuite sur la fiche documentaire du musée Ingres. @ Montauban, musée Ingres.

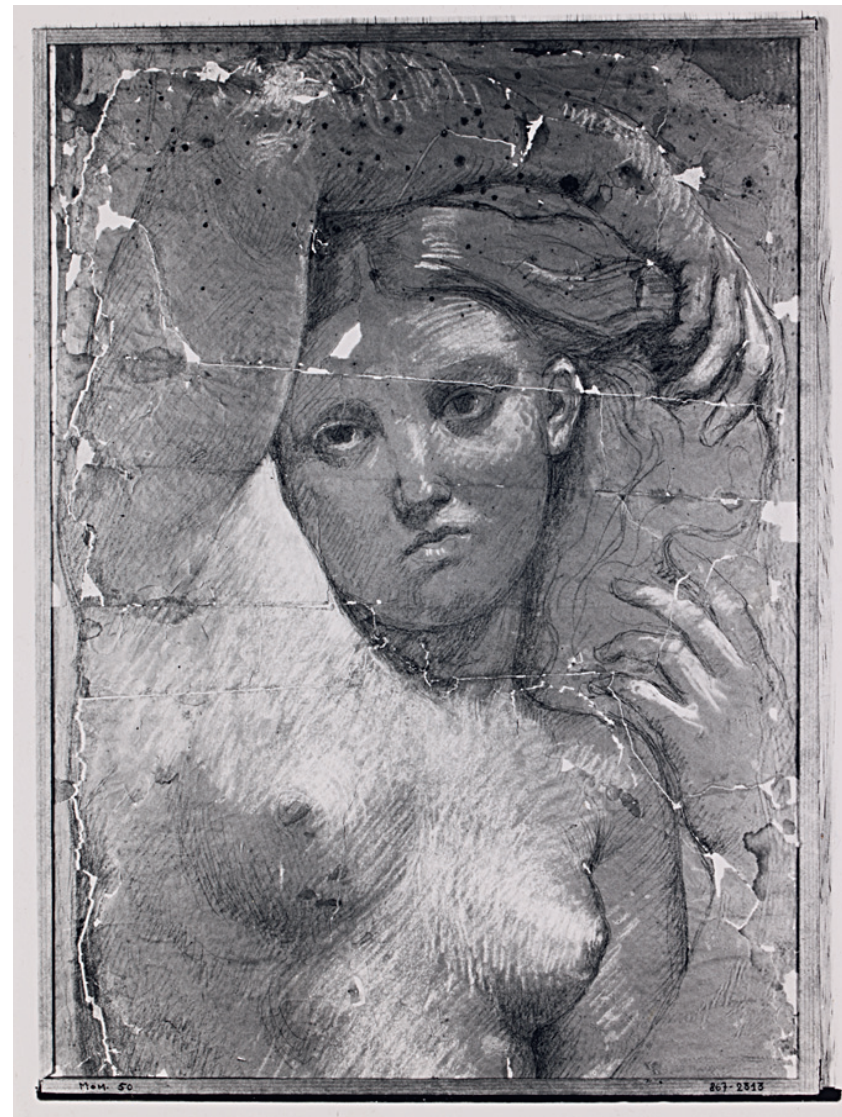

Fig. 4b. L'œuvre entre deux verres à Montauban avant sa restauration de 1992. On note le papier de bordage servant à tenir les verres et portant les numéros d'inventaire. @ Montauban, musée Ingres/ Guy Roumagnac. présentation et de garanties sérieuses contre le retour des causes de détérioration qu'il fut résolu de poursuivre l'effort muséographique ", écrit Jean Vergnet-Ruiz ${ }^{24}$. La prise en considération de l'environnement des œuvres est un point important : éviter la lumière, les variations hygrométriques et privilégier le roulement des œuvres exposées sont des composantes de l'aménagement du cabinet des dessins.

En juin 1949, les restaurations sont interrompues pour procéder à différents tests de produits et adhésifs utilisés notamment pour le blanchiment. Les matériaux constitutifs des montages et des futurs contenants sont aussi étudiés par des spécialistes en association avec le restaurateur.

\section{Produits et méthodes utilisés : un apport pour l'histoire de la restauration}

Les documents qui nous sont parvenus apportent des éléments de première importance dans le domaine de l'histoire de la restauration. Alors que les secrets d'ateliers perdurent souvent pour tout ce qui touche aux interventions sur les œuvres d'art, Léon Lepeltier est amené à décrire ses méthodes et procédés

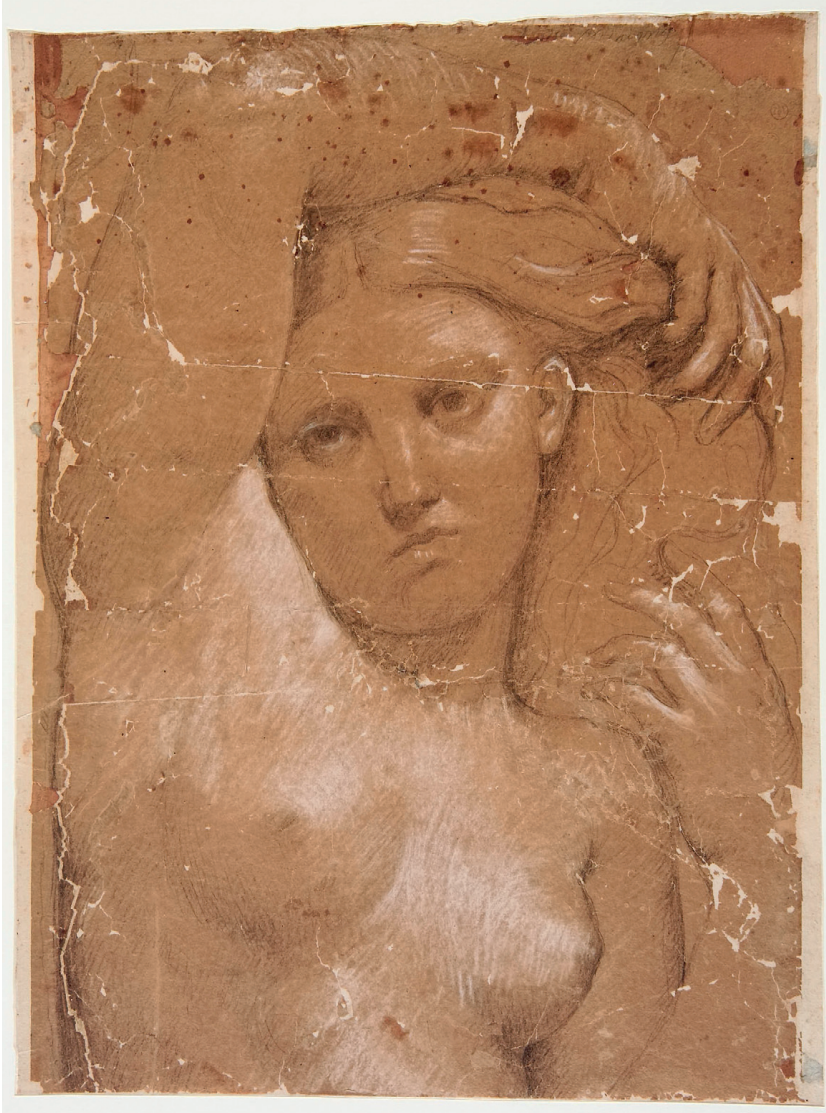

Fig. 4c. Après la restauration de 1992 (par Marie-Christine Enshaïan). (c) Montauban, musée Ingres/Guy Roumagnac. 
devant la «Commission de traitement et de montage des dessins d'Ingres » : « Pour leur [les papiers anciens] nettoyage il emploie soit les vapeurs de soufre, soit celles de chlore. Il opère à l'état humide et fait des sondages. (...) Il mouille à l'eau tiède complètement la feuille de papier, puis, pour les taches de rouille notamment, il emploie le permanganate à petite dose, la proportion résultant de plusieurs essais par petites touches. Pour parfaire l'effet du permanganate, il emploie l'acide nitrique ${ }^{25}$. $»$ En ce qui concerne les taches d'huile anciennes, il « les laisse, l'huile oxydée ne s'en allant pas. Les récentes, il les traite : il mouille complètement la feuille avec de l'alcool. En fait, il utilise la benzine ». Quant au collage, L. Lepeltier «ne semble pas s'être spécialement préoccupé de l'aseptisation des colles. Il dit les assainir avec de l'alcool camphréé26 ».

\section{Documenter en enquêtant}

\section{Le montage}

La question du montage occupe la plus grande partie des discussions, si bien qu'une sous-commission technique sur le montage se réunit le $1^{\text {er }}$ décembre 1948, avec pour membres : Jacqueline Bouchot-Saupique, Léon Lepeltier, Clémence Duprat, et deux scientifiques, Jacques Cogniard, chef du laboratoire de la fabrication des billets de la Banque de France $^{27}$ et M. de Saint-Rat, expert-technicien à la Bibliothèque nationale. La même année, les préconisations en matière de montage des œuvres sur papier sont données par Jacqueline Bouchot-Saupique : elle recommande l'emploi du passe-partout qui «semble le mieux répondre à la conservation et à la présentation des dessins d'Ingres ${ }^{28}$ ». Pourtant le passe-partout n'est pas complètement nouveau : il a été utilisé au Louvre dès l'époque de Villot ${ }^{29}$. On porte une attention particulière à l'adhésif employé qui «ne doit contenir aucun produit de conservation susceptible de tacher ou de détériorer de quelque autre façon le dessin. (...) Quand le papier est particulièrement fragile (notamment lorsqu'il s'agit de calques), on peut interposer une feuille d'acétate de cellulose ("Célastoid") qui sert de support transparent ${ }^{30}$ ».

Ainsi, les interrogations portent sur deux éléments essentiels : le papier et la colle. Un cahier des charges est rédigé par Jacques Cogniard pour obtenir une qualité de papier qui n'aura pas d'impact sur la conservation des œuvres : composition fibreuse, poids au $\mathrm{m}^{2}$, épaisseur, charge, résistance à la traction, au pliage et au collage sont pris en considération. Des échantillons de montages anciens de bonne qualité lui sont confiés pour analyse et pour servir de base à l'établissement de ce cahier des charges. Deux entreprises sont retenues pour les commandes de papiers et de cartons de montage : la maison Tochon-Lepage \& Cie est chargée de livrer les bristols sur lesquels les dessins seront montés, et les établissements E. Sevalle \& Cie, les cartons pour confectionner des portefeuilles $^{31}$.

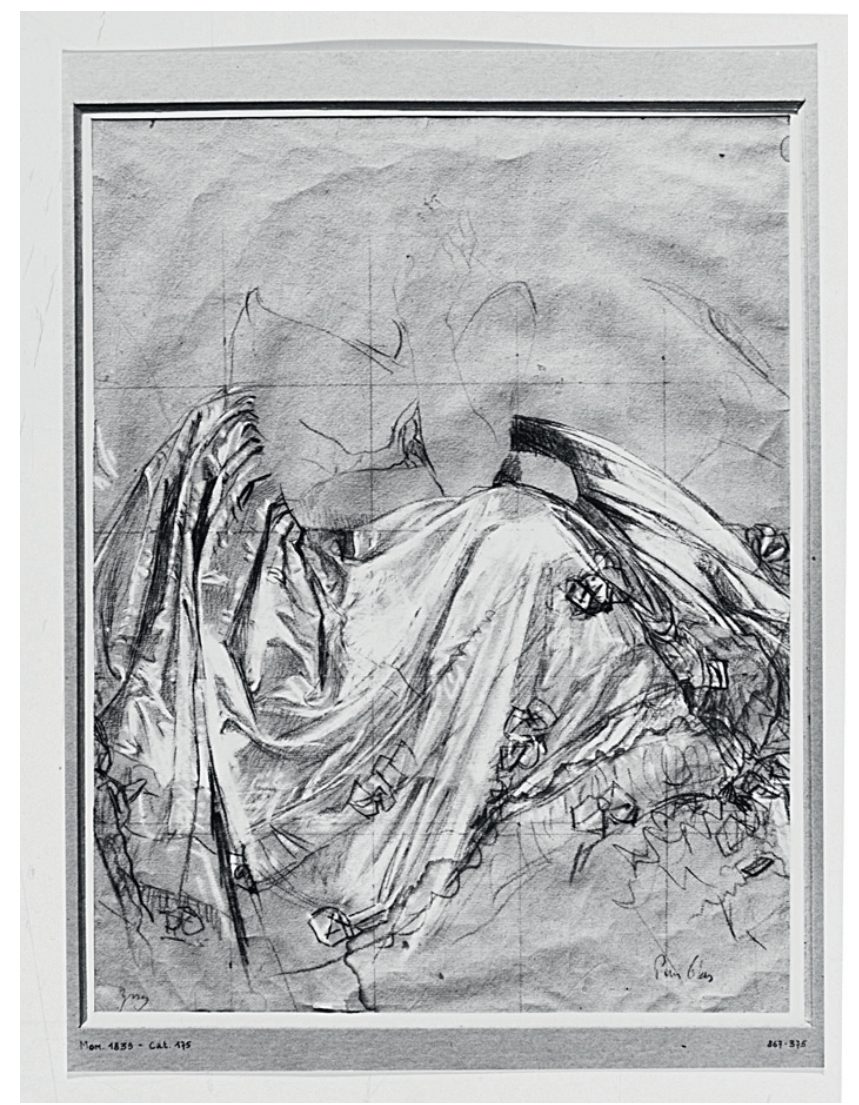

Fig. 5. Ingres, Étude pour le Portrait de la baronne Betty de Rothschild (MI 873.375). Photographie de Jaubert montrant l'ensemble du montage Lepeltier avec les numéros d'inventaire. (C) Montauban, musée Ingres.

\section{ICOM-News : "Le trait d'union de la grande famille des musées»}

Plus novatrices encore sont les recherches menées par la "Commission de traitement et de montage des dessins d'Ingres " qui demande une étude sur la composition chimique des papiers de montage. Elle consulte Agnes Mongan, conservateur au Fogg Art Museum de Harvard et spécialiste des dessins d'Ingres, pour recueillir son avis sur les montages des œuvres et pour connaître ses pratiques sur la collection dont elle a la charge ${ }^{32}$. Depuis 1928, le Fogg Art Museum possède en effet un atelier de restauration très renommé, le Straus Center for Conservation and Technical Studies, dirigé par Edward W. Forbes, qui comprend un chimiste, le Dr. Rutherford John Gettens, un responsable de la restauration, George Stout, et deux restauratrices, Evelyn Ehrlich et Minna Horwitz. Des questions très précises lui sont adressées en 1948, portant sur les traitements existant pour les taches (rouille, champignons, salissures), sur l'existence de normes pour les papiers et cartons de montage et sur les adhésifs utilisés, sur l'emploi de feuilles transparentes afin de protéger les dessins, ou encore sur les procédés permettant de consolider les papiers cassants. Cet entretien est publié dans le bulletin d'information de l'ICOM du $1^{\text {er }}$ février $1949^{33}$. 


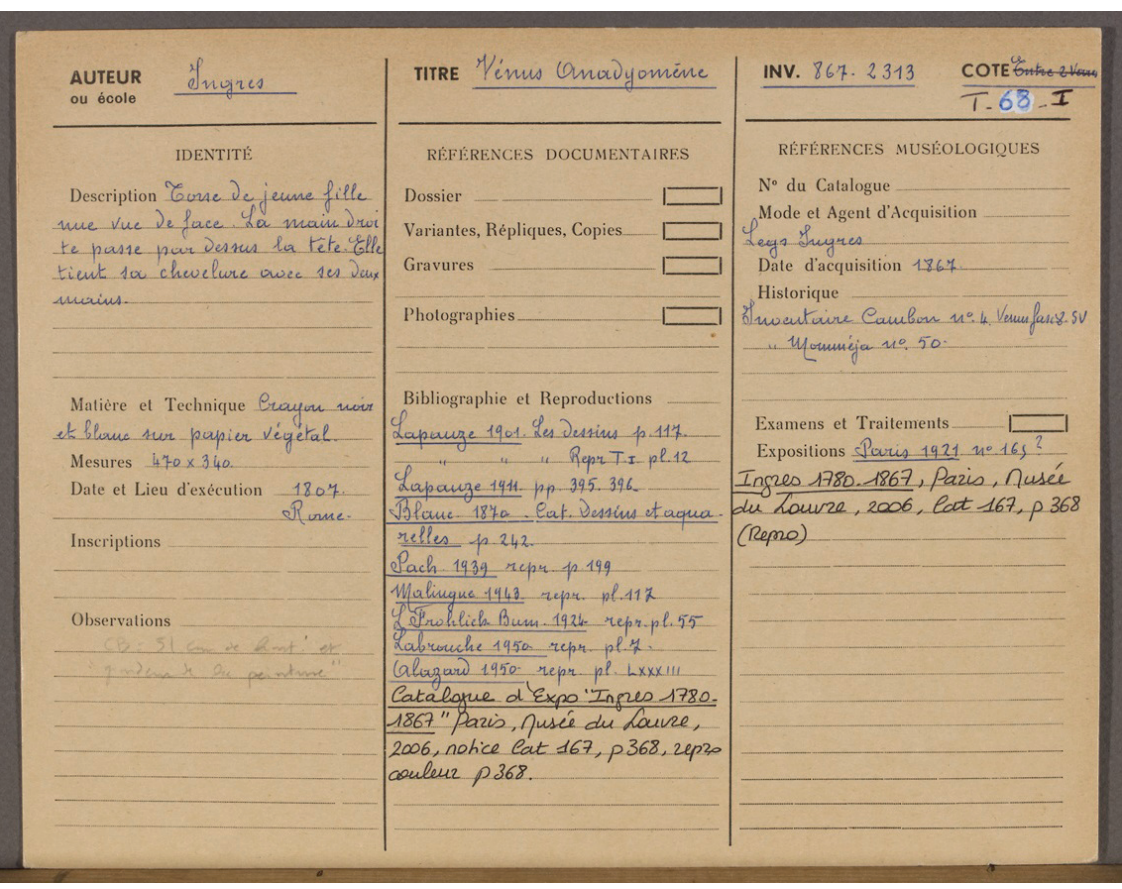

Fig. 6. Fiche établie par Daniel

Ternois pour Vénus Anadyomène.

(C) Montauban, musée Ingres,

archives.

travail accompli : Dessins d'Ingres $d u$ Musée de Montauban : première exposition temporaire. Une préface synthétique de Jean Vergnet-Ruiz résume les partis pris, des notices par Clémence Duprat accompagnent la présentation des dessins.

Cette campagne de restauration n'est que l'un des éléments du renouveau du musée. Elle met en évidence la qualité de la démarche documentaire : courriers, rapports, missions, enquêtes. On ne manquera pas d'insister sur l'apport encore nouveau, du moins pour des dessins, de la photographie

Aujourd'hui, le dossier Montauban de la série 20150044 aux Archives nationales conserve la mémoire de tous les essais de papier neutre pour le montage. La réalisation d'un carton offrant une qualité favorable à la conservation des œuvres tout en assurant leur sécurité (il est envisagé de créer un papier doté d'un filigrane spécifique, destiné aux musées et bibliothèques) n'a finalement pas abouti, ce qui a amené récemment le musée Ingres à se séparer progressivement de ses anciens cartons de montage pour les remplacer par des cartons neutres de la maison Canson, célèbre pour son papier «Ingres ».

En 1951, la nomination de Daniel Ternois à la tête du musée fait entrer ce dernier dans une nouvelle ère : tous les dessins sont photographiés dès leur retour au musée par Jaubert ${ }^{34}$. Ce sont donc ces photos qui peuvent donner encore aujourd'hui une idée de l'état des œuvres après les interventions de Lepeltier. Jaubert met en place une typologie de clichés laissant visible le passe-partout sur lequel figure le numéro d'inventaire (fig. 5). Les principes de Jaubert consistant à inclure les numéros d'inventaire du montage dans le cadrage du cliché seront d'ailleurs repris par ses différents successeurs, Résséguié et Roumagnac à ses débuts. Ces photographies sont conservées à Montauban, dans les dossiers d'œuvres. Elles complètent celles qui ont été prises par Séarl au Louvre avant restauration et qui ont été collées sur le verso des fiches descriptives réalisées pour chaque dessin, fiches encore en usage aujourd'hui (fig. 6). Les dessins sont exposés dans des vitrines spécifiquement conçues avec des supports à l'oblique ${ }^{35}$ (fig. 7). Enfin, un mince catalogue de quarante-deux pages, illustré de douze planches, paraît en 1951 à l'occasion de la présentation des œuvres au public, de juillet à octobre, qui met en valeur le comme témoin d'un état de l'œuvre et comme aide au suivi des restaurations. Ce chantier est resté dans le souvenir de ceux qui ont connu cette époque comme exemplaire de ce qui animait l'esprit de ces historiens de l'après-guerre. La personnalité rayonnante de Jean Vergnet-Ruiz y fut pour beaucoup. Ce dernier indiquait : "Dans l'évolution rapide des techniques muséographiques l'actuel cabinet de Montauban sera certainement dépassé quelque jour, mais il restera comme un des premiers et des meilleurs efforts de présentation d'un ensemble de dessins, depuis que ces techniques tendent à se répandre à travers le monde ${ }^{36}$. »

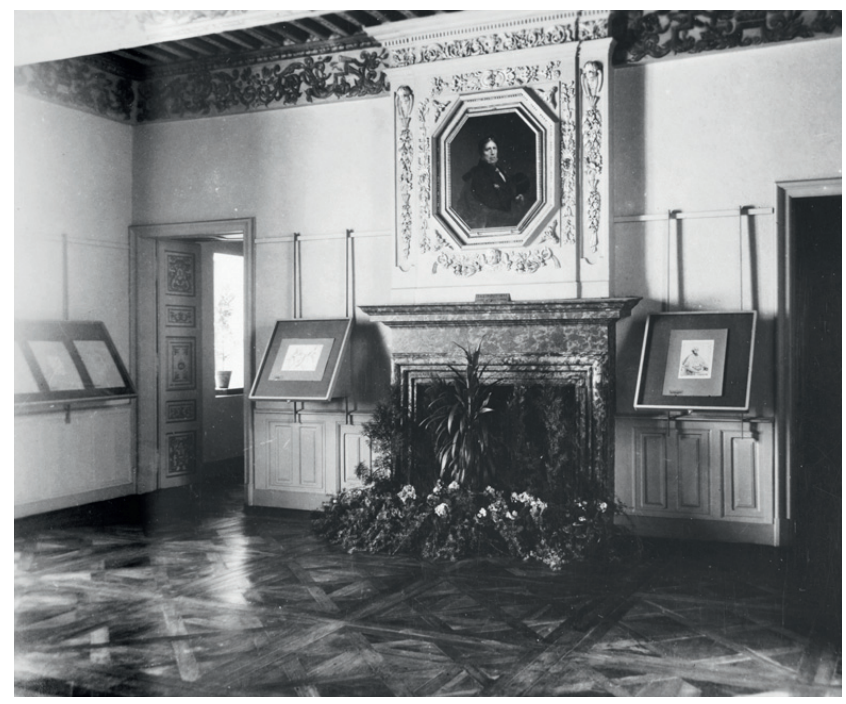

Fig. 7. La salle des dessins d'Ingres avec ses nouveaux meubles pupitres de présentation. (C) Montauban, musée Ingres, archives/ Jaubert. 
Notes

1. Coural, Desserrières, Raymond, 2008, p. $179-190$.

2. Archives nationales de France $(\mathrm{AnF})$ [archives des musées nationaux], O 30/662.

3. AnF, 20150467/262. Dépôt de La Poujade.

4. Félix Bouisset (1875-1960) occupe le poste de directeur du musée de Montauban de 1919 à 1951. Voir Bouisset F., 1926.

5. Exp. Montauban, 2011, p. 171-176.

6. Après « la drôle de guerre ", Jean Vergnet-Ruiz (1876-1972) est médecin chef de l'hôpital de Clermont. Collaborateur de Paul Jamot (1863-1939) au département des Peintures, il dirige en 1937 le laboratoire du Louvre. De 1945 à 1962, il est à la tête de l'Inspection générale des musées de Province. Voir Souvenirs, notes personnelles et témoignages, Paris, Les Presses artistiques, s. d.

7. AnF, 20150044/311. 15 décembre 1946.

8. Un premier inventaire sommaire est réalisé par une équipe du Louvre avant que Daniel Ternois, jeune conservateur nommé en 1950 , ne se lance à son tour dans l'opération qui débouchera sur l'inventaire complet et normalisé des dessins d'Ingres occupant aujourd'hui pas moins de dix registres.

9. Georges-Henri Rivière (1897-1985).

10. Jacqueline Bouchot-Saupique (18931975 ) est conservateur puis conservateur en chef du Cabinet des dessins de 1946 à 1963.

11. Léon Lepeltier (1877-1960). Fondation Custodia, 2012.

12. Émile Martin est alors président du Syndicat général de l'encadrement et de la dorure et travaille au musée du Louvre dès avant la guerre.

13. AnF, 20150044/312. 24 mars 1948.

14. Voir Achechova A., 2005. Nous remercions Aglaé Achechova-Renaudeau pour sa générosité. 1947.

15. AnF, 20150044/311. 22 octobre

16. Nous remercions Nathalie Volle qui a permis l'accès aux registres tenus entre 1942 et 1960 par Léon Lepeltier (Paris, archives privées). L'atelier de Léon Lepeltier se trouve alors 23, rue de la Villel'Evêque à Paris.

17. Exp. Paris, 1949. Les bénéfices sont reversés au musée Ingres pour la restauration des dessins. AnF, 20150044/311. Montauban, musée Ingres, MI 867.2592 et MI 867.277.

18. AnF, 20150044/312. Rapport d'inspection rédigé par Clémence Duprat et Georges-Henri Rivière, 1947.

19. AnF, 20150044/311. Rapport d'inspection de Clémence Duprat, 1948, p. 10 .

20. Paris, archives privées, carnets Lepeltier « Devis 1951-1952 ». Facture datée de mai-juillet 1951.
21. AnF, 20150044/311.

22. Montauban, musée Ingres, MI 867.2313.

23. Il a été possible depuis d'intervenir sur l'étude pour la Vénus Anadyomène dans le cadre d'une restauration longue, étalée sur plusieurs années entre 1992 et 2003.

24. Exp. Montauban, 1951, p. 4.

25. Voir les méthodes de blanchiment décrites sur les œuvres d'Ingres par le chimiste Rutherford John Gettens à Harvard : Smith T., 2012.

26. AnF, 20150044/312. Procès-verbal de la sous-commission technique sur le montage et le traitement des dessins, séance du $1^{\text {er }}$ décembre 1948 , p. $1-2$.

27. Jacques Cogniard (1903-?), chimiste, chef de laboratoire et membre du comité consultatif du laboratoire du musée du Louvre (communication écrite de Jean-Claude Camus, service des archives de la Banque de France, avril 2015).

28. AnF, 20150044/311. Rapport d'inspection de Clémence Duprat, 1948, p. 16.

29. Communication orale d'Irène Julier, atelier de montage du musée du Louvre (2014).

30. AnF, 20150044/311. Rapport d'inspection de Clémence Duprat, 1948, p. 16. Le Celastoid, un acétate de cellulose, n'est plus utilisé aujourd'hui, un autre film transparent (type Mylar) en Polyéthylène Terephthalate (PET) lui est préféré. Nous remercions Anne-Laurence Dupont, chargée de recherche au CNRS, Centre de Recherche sur la Conservation des Collections (CRCC), Paris

31. Établissements situés respectivement 46 , rue Vercingétorix et 56 , rue Saint-Sabin à Paris.

32. Agnes Mongan (1905-1996). 33. ICOM News, 1949 , p. 6-7. Les normes actuelles ISO 9706 et ISO 16245 diffèrent de la note de l'ICOM, tant en matière de composition que d'additifs. Le documen préconise des papiers $100 \%$ alpha-cellulose (sans hémicelluloses). Il s'agit donc d'un papier très pur, mais au coût élevé. Aujourd'hui, on accepte des papiers de pâte chimique blanchie ne contenant pas de lignine. Les produits antifongiques tels le thymol mentionné dans le document comme additif à la colle sont à manipuler avec précaution, car ils sont toxiques pour la santé (communication écrite d'AnneLaurence Dupont, CRCC, 15 juin 2015).

34. Un sondage effectué dans les dossiers d'œuvres fait apparaître, grâce à des annotations au dos des clichés, que le photographe avec lequel Daniel Ternois a travaillé principalement est M. Jaubert dont le tampon apparaît au dos des images, parfois accompagné d'une inscription de Daniel Ternois.

35. Un mobilier spécifique est créé par J. Barre, décorateur, 3 rue Sivel à Paris, pour présenter les dessins : pupitres simples et pupitres double face.

36. Exp. Montauban, 1951, p. 5

\section{Bibliographie}

Bouisset F., 1926, Le Musée Ingres, Montauban.

Coural N., Desserrières L., Raymond F., 2008, « Lille, Paris, Saint-Quentin, Montauban. Conservation et présentation des dessins aux XIX ${ }^{\mathrm{e}}$ et $\mathrm{XX}^{\mathrm{e}}$ siècles ", Technè, $\mathrm{n}^{\circ} 27-28$, p. 179190.

ICOM News. Bulletin d'information publié par le Conseil International des Musées, vol. 2, $\mathrm{n}^{\circ} 1$, février 1949 .

Exp. Montauban, 1951 : Les Dessins d'Ingres du musée de Montauban : première exposition temporaire [Exposition. Montauban, musée Ingres, 1951].

Exp. Montauban, 2011 : Ingres, secrets de dessins [Exposition. Montauban, musée Ingres, 2011], Viguier-Dutheil F., Guicharnaud H., Duval A., Cailleteau M, et Langlois J.

Exp. Montauban, 2013: Quoi de neuf au musée ? Acte I " 10 ans de restauration de dessins d'Ingres [Exposition. Montauban, musée Ingres, 2013].

Exp. Paris, 1949 : Exposition Ingres au profit du musée Ingres de Montauban [Exposition. Paris, Galerie André Weil, 1949].

Fondation Custodia, 2012, « Léon Lepeltier », Les Marques de Collections de Dessins et d'Estampes [En ligne], mis en ligne en mars 2010, consulté le 14 juin 2015. URL : www.marquesdecollections.fr.

Plenderleith H.J., 1934 et 1936 , «La Conservation des estampes, dessins et manuscrits ", Mouseion, XXIX-XXX, p. 81-90 et XXXIIIXXXIV, p. 199-226.

Smith T., 2012, "An Evaluation of Historical Bleaching with Chlorine Dioxide Gas, Sodium Hypochlorite, and Chloramine $\mathrm{T}$ at the Fogg Museum", Restaurator, vol. 33, p. 240-273.

Vigne G., 1995, Dessins d'Ingres : catalogue raisonné des dessins de Montauban, Gallimard, RMN, Paris.

\section{Documents inédits}

Achechova, A., 2005, La Campagne de restauration et de montage des dessins d'Ingres au musée du Louvre (1948-1960), Dossier DRA, École du Louvre, Paris. 